\title{
All-fiber-integrated soliton-similariton laser with in-line fiber filter
}

\author{
Zuxing Zhang, ${ }^{1, *}$ B. Öktem, ${ }^{2}$ and F. Ö. Ilday ${ }^{1}$ \\ ${ }^{1}$ Department of Physics, Bilkent University, 06800 Ankara, Turkey \\ ${ }^{2}$ Institute of Materials Science and Nanotechnology, Bilkent University, 06800 Ankara, Turkey \\ *Corresponding author: zhang@fen.bilkent.edu.tr
}

Received June 8, 2012; revised July 12, 2012; accepted July 12, 2012;

posted July 13, 2012 (Doc. ID 170231); published August 16, 2012

\begin{abstract}
We demonstrate an all-fiber-integrated Er-doped fiber laser operating in the soliton-similariton mode-locking regime. In the similariton part of the cavity, a self-similarly evolving parabolic pulse with highly linear chirp propagates in the presence of normal dispersion. Following an in-line fiber-based birefringent filter, the pulse evolves into a soliton in the part of the cavity with anomalous dispersion. The similariton and the soliton pulses are dechirped to 75.5 and $167.2 \mathrm{fs}$, respectively, outside of the cavity. Mode-locked operation is very robust, owing to the influence of the two similariton and soliton attractors that predominate each half of the laser cavity. The experimental results are supported with numerical simulations, which provide good agreement. @ 2012 Optical Society of America

OCIS codes: $\quad 060.2320,140.7090,190.4370,320.7090$.
\end{abstract}

Passively mode-locked fiber lasers have been intensively investigated due to their potential in realizing reliable and cost-effective ultrafast light sources [1]. Four distinct regimes of pulse shaping are well known for passively mode-locked lasers, including soliton [2], stretched-pulse [ㄱ] , similariton [4] ], and all-normal dispersion regimes [ㅁ] . More recently, a fifth regime, combining the similariton and soliton regimes, has been identified, namely the soliton-similariton laser []. A A conventional soliton formed by the balance of self-phase modulation and negative group-velocity dispersion (GVD) is chirp free and propagates unchanged, but its pulse energy is limited to a low level. Stretched-pulse or dispersion-managed soliton operation exists for net anomalous or small normal GVD and allows generation of femtosecond pulses with up to several nanojoule energies. Oscillators supporting selfsimilar pulses (similaritons) [ㄴ,7] are able to tolerate strong nonlinearity without wave breaking, allowing scaling up of the pulse energy to $10 \mathrm{~nJ}$ in single-mode fiber (SMF) [8]. Additionally, dissipative solitons in all-normaldispersion lasers balance nonlinear phases by spectral filtering of a highly chirped pulse in the laser cavity, avoiding wave breaking [5]. The soliton-similariton laser supports two distinct types of nonlinear shapepreserving pulses within the same cavity: solitons and similaritons, each of which acts as attractors for the pulse shaping dynamics. The soliton-similariton laser also has the interesting feature of tolerating the strongest nonlinear effects observed in any laser to date [6]. This aspect can be quantified through the nonlinear phase shift that the pulse can tolerate or through the amount of spectral broadening occurring within the cavity.

Realization of compact, high-stability, and alignmentfree operation of fiber lasers is highly desirable. Dispersion control of the laser cavity can be easily implemented using fibers with normal GVD and anomalous GVD above $1.3 \mu \mathrm{m}$ (zero dispersion point of fused silica). In the similariton pulse regime, spectral filtering plays a crucial role due to the extreme spectral broadening that an amplifier similariton experiences [7]. The common approach is to use an interference or birefringent filter. However, these filters are bulk components, and filter bandwidth is not adjustable. Mode-locked operation using a Lyot birefringence filter, implemented in fiber by combining an in-line polarizer followed by a segment of polarizationmaintaining (PM) fiber, was recently demonstrated [9]. In addition to its simplicity, the filter bandwidth is adjustable through the length of the PM fiber.

Here, we report, for the first time to the best of our knowledge, an all-fiber-integrated soliton-similariton laser. Filtering is achieved using an in-line PM fiber-based birefringence fiber filter. We show that the pulse propagates self-similarly in the gain fiber under normal dispersion with highly linear chirp. Following spectral filtering, the pulse gradually evolves into a soliton in the rest of the cavity, which has anomalous dispersion. Mode-locked operation is notably robust and stable, which we attribute to the dominance of the similariton and soliton attractors within the laser cavity.

The experimental setup is illustrated in Fig. 1(a). The gain fiber is a $1.2 \mathrm{~m}$ long Er-doped fiber (EDF), followed by a $90: 10$ coupler and isolator hybrid with $10 \%$ port. Up to $550 \mathrm{~mW}$ of $980 \mathrm{~nm}$ pump light is delivered to the cavity via a 980/1550 nm wavelength-division multiplexer (WDM) from a laser diode. The EDF has mode field diameter of $4.9 \mu \mathrm{m}$, NA of 0.29 and GVD of $79.4 \mathrm{fs}^{2} / \mathrm{mm}$ at $1.55 \mu \mathrm{m}$. An in-line polarizer, sandwiched with two polarization controllers (PCs), is used to convert nonlinear polarization rotation to amplitude modulation, initiating and stabilizing mode-locked operation [10]. Length of SMF from the EDF to the polarizer input is $0.66 \mathrm{~m}$. The short, the PM lead fiber of the in-fiber polarizer is spliced to another section of PM fiber, whose length can be selected to achieve the desired filter bandwidth. The axes of the two PM fibers are spliced at a $45^{\circ}$ angle, thereby coupling light into both axes of the PM fiber. The calculated relation between filter bandwidth and the PM fiber length is shown in Fig. 1(b), and the scattering symbols correspond to measurements. A second 10\% coupler with short SMF pigtails connects the PM fiber to the signal input port of the WDM, fulfilling an all-fiber ring cavity 

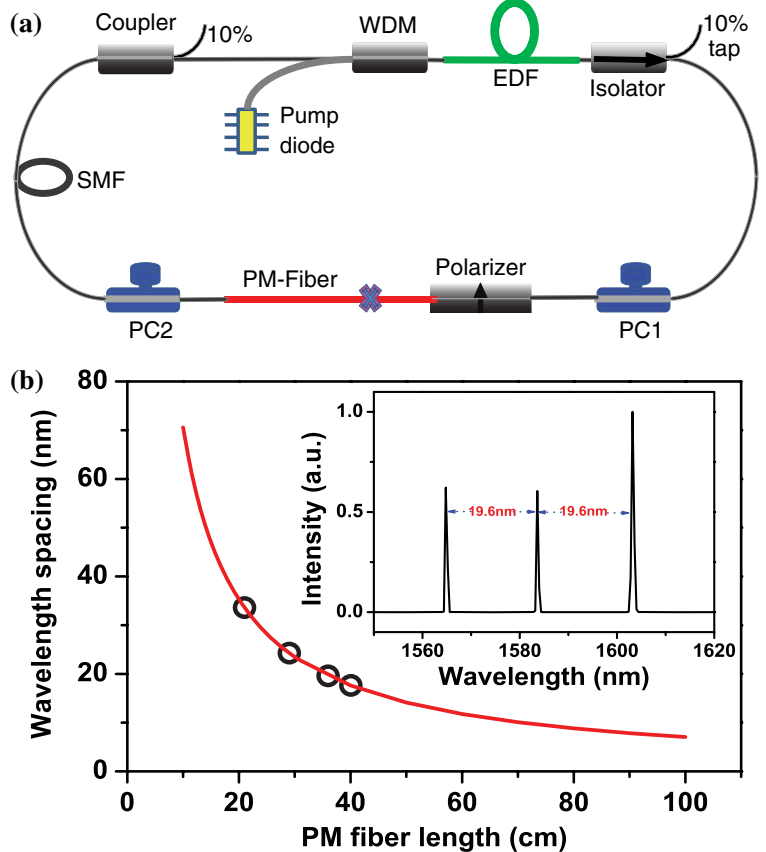

Fig. 1. (Color online) (a) Schematic diagram of the experimental setup of the all-fiber-integrated soliton-similariton laser and (b) calculated relation between filter bandwidth and PM fiber length; symbols correspond to experimental data. Inset, optical spectrum of CW operation with wavelength spacing of $19.6 \mathrm{~nm}$, corresponding to the filter bandwidth.

configuration. The net GVD of the laser cavity was calculated to be about $0.018 \mathrm{ps}^{2}$.

In order to understand the effect of filter bandwidth on the laser output, we varied the PM fiber length from 21 to $40 \mathrm{~cm}$. Mode-locked operation, as detailed below, could always be attained within this range. Here we report the optimal results, which are obtained for a PM fiber length of $36 \mathrm{~cm}$. This length corresponds to $19.6 \mathrm{~nm}$ bandwidth, which is confirmed by the $\mathrm{CW}$ operation of the laser [inset of Fig. 1(b)]. Self-starting mode-locked operation is achieved readily and very stably by adjustment of the PCs. Threshold pump power for mode locking is $360 \mathrm{~mW}$. The mode-locked laser produces a stable pulse train with $51.1 \mathrm{MHz}$ of repetition rate. The output power from the coupler after the gain fiber can reach $31.6 \mathrm{~mW}$, corresponding to an intracavity pulse energy of $6.18 \mathrm{~nJ}$. The characterizations of the laser output are given in Fig. 2. Meanwhile, numerical simulations were conducted to provide a guidance for the experiments and deeper understanding of laser dynamics. The model is based on a nonlinear Schrödinger equation, generalized to include higher-order dispersion, Raman scattering, gain with saturation and bandwidth filtering, saturable absorption, and the bandpass filter. The details of the model can be found in [6].

Figure 2(a) shows the calculated evolution of the spectral bandwidth and pulse duration as a function of position along the cavity: The broadening in both spectral and time domains takes place predominantly within the normal-GVD EDF, while in the SMF after the filter the spectral width and pulse duration undergo smaller adjustments. Figures 2(b) and 2(c) show the experimental optical spectra measured before and after the gain fiber,
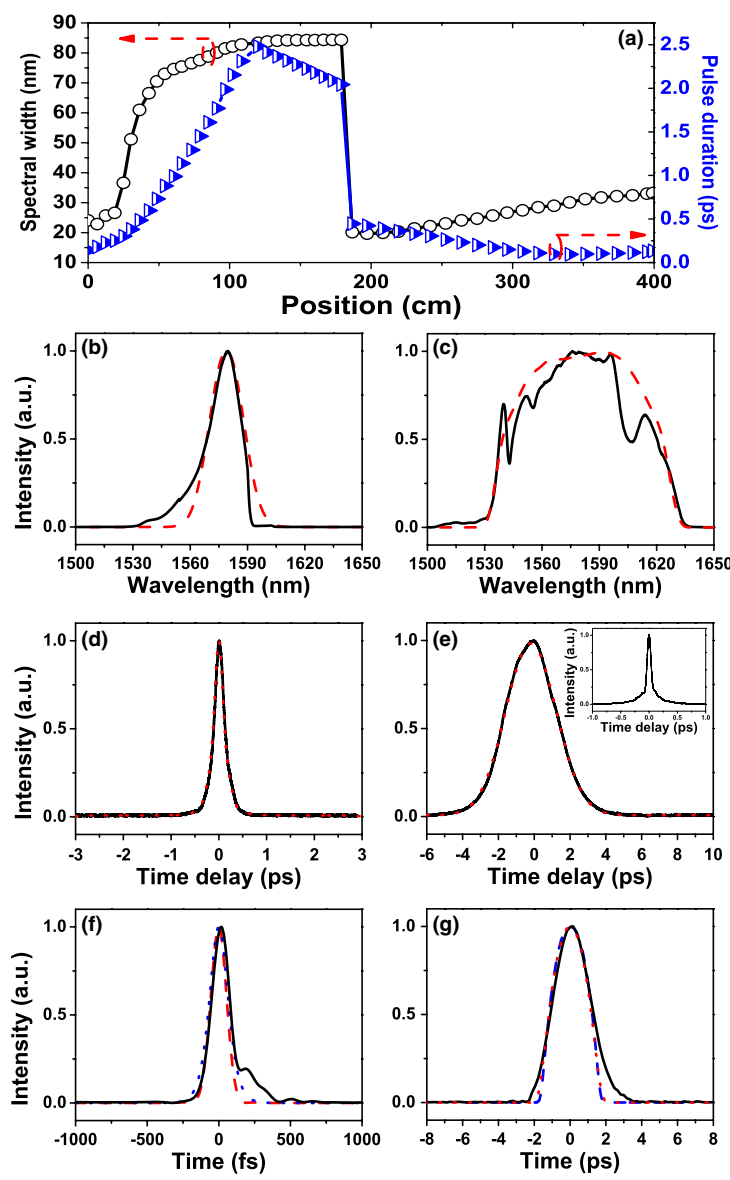

Fig. 2. (Color online) (a) Evolution of the spectral and temporal width of the pulse as a function of position along the cavity. Experimental (solid line) and simulated (dashed line) optical spectrum with linear vertical axis of (b) the soliton pulse, measured from the $10 \%$ coupler before the gain fiber and (c) the similariton pulse, measured from the $10 \%$ coupler after the gain fiber. Autocorrelation (solid line) of the (d) soliton and (e) similariton pulses with PICASO fitting (dotted line). (Inset) Autocorrelation of the similariton pulse after compression. Pulse shape (solid line) retrieved using the PICASO algorithm and simulated temporal intensity profile (dashed line) for the (f) soliton and (g) similariton pulses with $\operatorname{sech}^{2}(t)$ and parabolic fitting (dotted line), respectively.

respectively, along with the corresponding simulated spectra. The measured FWHM values for the optical spectra are $19.4 \mathrm{~nm}$, consistent with the filter bandwidth, and $82.6 \mathrm{~nm}$, respectively. The spectral breathing ratio is 4.3. The autocorrelation traces from the two output ports are plotted in Figs. 2(d) and 2(e). The PICASO algorithm [11] was used to infer the pulse shape from the measured autocorrelation and spectrum. The measured pulse width for the soliton pulse is 167.2 fs. The PICASO-retrieved pulse shape and numerically simulated temporal intensity profiles, as shown in Fig. 2(f), match well a hyperbolic secant fitting, as expected for a soliton. The measured pulse width for the similariton pulse is $2.2 \mathrm{ps}$. The PICASO-retrieved pulse shape and simulated temporal intensity profiles, as shown in Fig. 2(g), match well a parabolic pulse shape, as expected. The similariton pulses were compressed in a $1.8 \mathrm{~m}$ long segment of SMF to 75.5 fs. The zero-phase Fourier-transform 
calculation yields a theoretical lower limit of $62 \mathrm{fs}$, indicating that the uncompressed pulses are have largely linear chirp, as required for a similariton.

From this set of measurements, the evolution of the pulses within the cavity clearly emerges. The pulses before and after the gain fiber have hyperbolic secant shapes, propagating as solitons, and parabolic shapes, propagating as similaritons, respectively. The similariton propagates $[\underline{12}, 13]$ in the normal-GVD EDF without pulse breakup despite its high energy of $6.2 \mathrm{~nJ}$, owing to seeding of the evolution with a narrow spectrum. Upon filtering, the pulse enter the SMF, where soliton dynamics shape the pulse. The pulse assumes a hyperbolic secant temporal profile and stabilizes in the temporal and spectral width, before entering the gain fiber, confirming the soliton nature of propagation in this part of the cavity.

The fundamental repetition rate of the laser is $51.1 \mathrm{MHz}$, and the RF spectrum with $1 \mathrm{GHz}$ scanning range and $1 \mathrm{kHz}$ resolution bandwidth is shown in the inset of Fig. 3(a). This oscillator has excellent stability both in the short and long term. The laser continues mode-locking operation uninterrupted for at least several weeks. RF spectrum with $1 \mathrm{kHz}$ scanning range and $10 \mathrm{~Hz}$ resolution bandwidth shows $104 \mathrm{~dB}$ sideband suppression ratio, including the sidebands at 50 and $100 \mathrm{~Hz}$, arising from the power line fluctuations [Fig. 3(a)]. The high-level of short-term stability indicated by the $\mathrm{RF}$ spectrum is confirmed by relative intensity noise (RIN) measurements [Fig. 3(b)]. These measurements yield an integrated RIN value of $0.01 \%$ over the $3 \mathrm{~Hz}-$ $250 \mathrm{kHz}$ range, corresponding to better-than-usual performance compared to conventional fiber lasers [14]. We believe noise performance is currently limited by the driver electronics for the pump laser rather than the fiber laser cavity dynamics. We attribute the superior noise performance of the laser to the strong attractors dominating the pulse shaping.

In conclusion, we have demonstrated what is to our knowledge the first all-fiber-integrated soliton-similariton laser, utilizing a PM fiber section inserted into laser cavity acting as a filter. The existence of similariton and soliton pulses are experimentally confirmed in the corresponding parts of the cavity. In addition, we obtain pulses shorter and more energetic than in [6]. The experimental results are supported by numerical simulations with very good agreement. The mode-locked operation is very robust under the influence of strong nonlinear attractors, namely
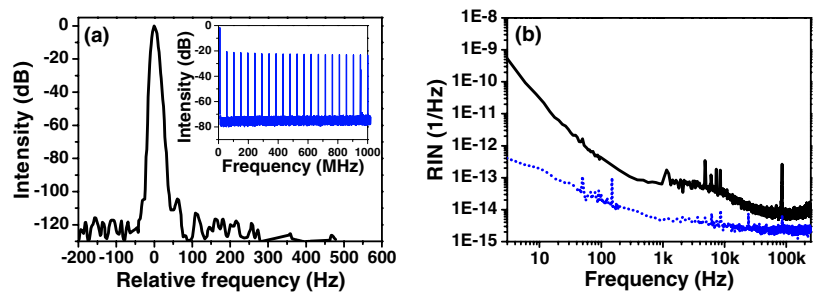

Fig. 3. (Color online) (a) Measured RF spectrum with $1 \mathrm{kHz}$ span and $1 \mathrm{~Hz}$ resolution bandwidth, with central frequency shifted to zero for clarity. Inset, RF spectrum with $1 \mathrm{GHz}$ span and $1 \mathrm{kHz}$ resolution bandwidth. (b) Measured RIN (solid line) along with the noise floor (dotted line).

the soliton and the similariton solutions. We expect this highly practical, low-noise oscillator to find various applications, particularly as seed sources for Er-fiber amplifiers.

This work was supported by TÜBİTAK under grant no. 209T058 and European Union (EU) FP7 CROSSTRAP Project (grant no. 244068).

\section{References}

1. W. H. Renninger, A. Chong, and F. W. Wise, IEEE J. Sel. Top. Quantum Electron. 18, 389 (2012).

2. I. N. Duling III, Electron. Lett. 27, 544 (1991).

3. K. Tamura, E. P. Ippen, H. A. Haus, and L. E. Nelson, Opt. Lett. 18, 1080 (1993).

4. F. Ö. Ilday, J. R. Buckley, W. G. Clark, and F. W. Wise, Phys. Rev. Lett. 92, 213902 (2004).

5. A. Chong, J. Buckley, W. Renninger, and F. W. Wise, Opt. Express 14, 10095 (2006).

6. B. Oktem, C. Ülgüdür, and F.Ö. Ilday, Nat. Photon. 4, 307 (2010).

7. W. H. Renninger, A. Chong, and F. W. Wise, Phys. Rev. A 82, 021805 (2010).

8. J. R. Buckley, F. Ö. Ilday, and F. W. Wise, Opt. Lett. 30, 1888 (2005).

9. K. Özgören and F. Ö. Ilday, Opt. Lett. 35, 1296 (2010).

10. K. M. Spaulding, D. H. Yong, A. D. Kim, and J. N. Kutz, J. Opt. Soc. Am. B 19, 1045 (2002).

11. J. W. Nicholson, J. Jasapara, W. Rudolph, F. G. Omenetto, and A. J. Taylor, Opt. Lett. 24, 1774 (1999).

12. V. I. Kruglov, A. C. Peacock, J. M. Dudley, and J. D. Harvey, Opt. Lett. 25, 1753 (2000).

13. M. E. Fermann, V. I. Kruglov, B. C. Thomsen, J. M. Dudley, and J. D. Harvey, Phys. Rev. Lett. 84, 6010 (2000).

14. I. L. Budunolu, C. Ülgüdür, B. Oktem, and F. Ö. Ilday, Opt. Lett. 34, 2516 (2009). 